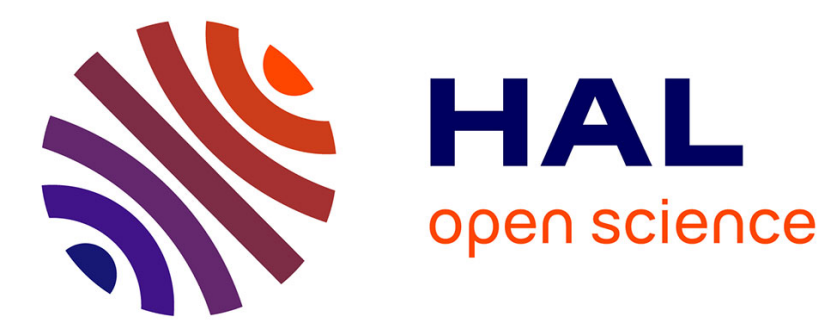

\title{
B hadron decays and resonances at D0
}

\author{
D. Gelé
}

\section{To cite this version:}

D. Gelé. B hadron decays and resonances at D0. 14th International Workshop on Deep Inelastic Scattering (DIS 2006), Apr 2006, Tsukuba, Japan. in2p3-00126154

\section{HAL Id: in2p3-00126154 https://hal.in2p3.fr/in2p3-00126154}

Submitted on 23 Jan 2007

HAL is a multi-disciplinary open access archive for the deposit and dissemination of scientific research documents, whether they are published or not. The documents may come from teaching and research institutions in France or abroad, or from public or private research centers.
L'archive ouverte pluridisciplinaire HAL, est destinée au dépôt et à la diffusion de documents scientifiques de niveau recherche, publiés ou non, émanant des établissements d'enseignement et de recherche français ou étrangers, des laboratoires publics ou privés. 


\title{
$B$ HADRON DECAYS AND RESONANCES AT D0
}

\author{
DENIS GELÉ \\ Institut Pluridisciplinaire Hubert Curien, \\ Département Recherches Subatomiques, \\ Strasbourg, FRANCE \\ E-mail: gele@in2p3.fr
}

Using D0 data collected at Tevatron collider since april 2002, new measurements of excited $B_{d}^{0}$ and $B_{s}^{0}$ mesons decays properties are presented. Searches for rare $B_{s}^{0}$ decay channels are summarized with expected limits on their production rate. The study of the $B_{s}^{0} \rightarrow J / \psi \phi$ decay is performed to obtain the $B_{s}^{0}$ lifetime as well as some others characteristics of the $\left(B_{s}^{0}, \bar{B}_{s}^{0}\right)$ system.

Theoretical quark models relating to the spectroscopy of $B_{d}^{0}$ (and $B_{s}^{0}$ mesons $)^{1}$ predicts the existence of two excited narrow $P$ states denoted $B_{1}$ and $B_{2}^{*}$ ( $B_{s 1}$ and $B_{s 2}^{*}$ respectively) which have never been observed unambigously. Those states should decay through a $D$ wave $(L=2)$ with a small width of around $10 \mathrm{MeV}$. Using an integrated luminosity of $1 \mathrm{fb}^{-1}$, the D0 experiment studied the reconstructed $B_{1}$ and $B_{2}^{*}$ states through the following decays: $\left(B_{1}^{0} \rightarrow B^{*+} \pi^{-}, B^{*+} \rightarrow B^{+} \gamma\right),\left(B_{2}^{0 *} \rightarrow B^{*+} \pi^{-}, B^{*+} \rightarrow\right.$ $\left.B^{+} \gamma\right)$ and $\left(B_{2}^{0 *} \rightarrow B^{+} \pi^{-}\right)$. The final decay product of the $B^{*+}$ meson is a $B^{+}$with a release of an undetected photon of $45.78 \pm 0.35 \mathrm{MeV}$. The $B^{+}$ mesons are reconstructed in the exclusive decay: $B^{+} \rightarrow J / \psi K^{+}$with $J / \psi$ decaying to $\mu^{+} \mu^{-}$. The selection of the $16219 \pm 180 B^{+}$candidates is based on the presence of two identified muons correctly associated with a kaon track. By means of a likelihood ratio method, the $B_{J}$ selection is achieved by requiring an additionnal track originating from the primary vertex with a correct pion charge correlation . The distribution of the mass difference given by $\Delta M=M\left(B^{+} \pi^{-}\right)-M\left(B^{+}\right)$and fitted with a binned likelihood function (described by the convolution of a relativistic Breit-Wigner with the experimental resolution on $\Delta M)$ can be interpreted in terms of $B_{J}$ transitions as illustrated in Fig. 1 . The $B_{1}$ and $B_{2}^{*}$ mesons are observed for the first time as two separate states and their masse and their average width (set equal in the fit) were measured to be: $M\left(B_{1}\right)=5720.8 \pm 2.5($ stat $) \pm$ 
5.3(sys) $M e V, M\left(B_{2}^{*}\right)-M\left(B_{1}\right)=25.2 \pm 3.0 \pm 1.1 M e V, \Gamma\left(B_{1}\right)=\Gamma\left(B_{2}^{*}\right)=$ $6.6 \pm 5.3 \pm 4.2 \mathrm{MeV}$. The branching ratio of $B_{2}^{*}$ to the excited state $B^{*}$ was fitted as $\operatorname{Br}\left(B_{2}^{*} \rightarrow B^{*} \pi\right) / B r\left(B_{2}^{*} \rightarrow B^{(*)} \pi\right)=0.513 \pm 0.092 \pm 0.115$. The branching ratio of the $B_{J}$ sample in the state $B_{1}$ was measured as $B r\left(B_{1} \rightarrow B^{*} \pi\right) / B r\left(B_{J} \rightarrow B^{(*)} \pi\right)=0.545 \pm 0.064 \pm 0.071$. Finally, the $B_{J}$ is measured as a fraction of the $B^{+}$rate with $0.165 \pm 0.024 \pm 0.028$.

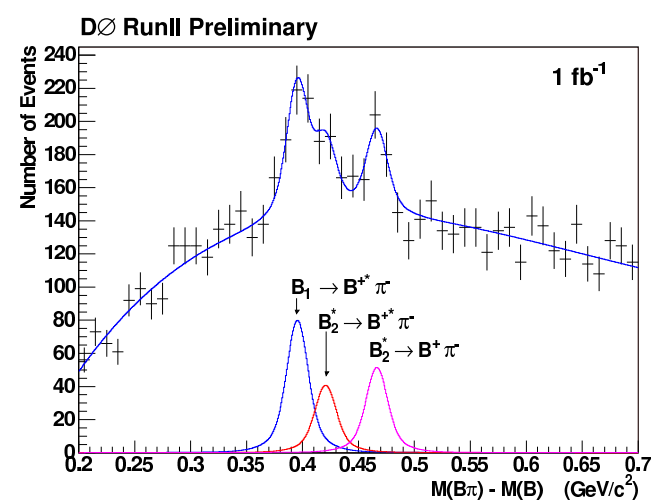

Figure 1. Mass difference $\Delta M=M\left(B^{+} \pi^{-}\right)-M\left(B^{+}\right)$for exclusive $B$ decays with the corresponding 3 peaks shown separately.

The analysis of $B_{s J}$ states decaying to $B^{+} K^{-}$is very similar to the previous one, changing the kaon mass assignation to the additional track. Fig. 2 shows the first direct observation of the $B_{s 2}^{*}$ signal with a significance greater than 5 and a fitted mass $M\left(B_{s 2}^{*}\right)=5839.1 \pm 1.4 \pm 1.5 \mathrm{MeV}$. The absence of the $B_{s 1}$ signal is due to the short mass difference between the $B_{s 2}^{*}$ signal and the kinematical constraint $M\left(B_{s 1}\right)<M\left(B^{+*}\right)-M\left(K^{-}\right)$.

The decay $B_{s}^{0} \rightarrow \mu^{+} \mu^{-}(\phi)$ is an FCNC process forbidden in the Standard Model at tree level which proceeds at a very low rate in higher order diagrams $\left(B r_{S M} \sim 10^{-9}\right)$. D0 performs a blind analysis with $700 p b^{-1}$ based on the search for a secondary 3D-vertex built from two oppositely charged tracks and some requirements on discriminating variables. The signal was optimized with a random grid search method. In the $\mu^{+} \mu^{-}$invariant mass signal region 4 events compatible with the expected background were found after selection . Using the $B^{+} \rightarrow J / \psi K^{+}$process as a normalisation channel, D0 obtains the branching ratio limit $\operatorname{Br}\left(B_{s}^{0} \rightarrow \mu^{+} \mu^{-}\right)<2.3 .10^{-7}$ at $95 \%$ CL. For the $B_{s}^{0} \rightarrow \mu^{+} \mu^{-} \phi$ decay channel, the limit on the branching ratio is $4.1 \cdot 10^{-6}$ at $95 \%$ CL for $300 p b^{-1}$. 


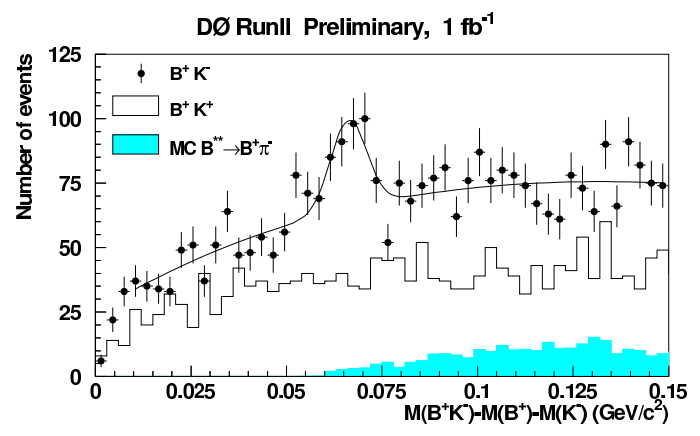

Figure 2. Mass difference $\Delta M=M\left(B^{+} K^{-}\right)-M\left(B^{+}\right)-M\left(K^{-}\right)$for exclusive $B$ decay. The histogram shows the mass difference for $B^{+} K^{+}$. The solid histogram represents the MC distibution of the decay $B^{* *} \rightarrow B^{(*)} \pi$ where the $\pi$ is misidentified as a kaon.

In the Standard Model, the mass eigenstates of $B_{s}^{0}$ mesons $\left(B_{s}^{H}\right.$ and $\left.B_{s}^{L}\right)$ are linear combinations of flavor eigenstates and also approximately $\mathrm{CP}$ eigenstates. Defining $\Delta m=m_{H}-m_{L}, \Delta \Gamma=\Gamma_{L}-\Gamma_{H}$ and $\Gamma=\left(\Gamma_{H}+\Gamma_{L}\right) / 2, B_{s}^{0}$ mesons are produced in an equal mixture of $B_{H}$ and $B_{L}$ and their decay length is described by $\exp \left(-\Gamma_{H} t\right)+\exp \left(-\Gamma_{L} t\right)$ instead of $\exp (-\Gamma t)$ (assuming a single lifetime). The first analysis reconstructs the semileptonic decay channel $B_{s}^{0} \rightarrow D_{s}^{-} \mu^{+} \nu X, D_{s}^{-} \rightarrow \phi \pi^{-}, \phi \rightarrow K^{+} K^{-}$using a $400 p b^{-1}$ data sample. The pseudo-proper decay length defined as $L_{x y} m\left(B_{s}^{0}\right) / P_{T}\left(D_{s}^{-} \mu^{+}\right)$ (where $L_{x y}$ is the transverse decay length) is fitted by means of an unbinned maximum likelihood method under the assumption of a singleexponential decay and leads to the $B_{s}$ lifetime results (Fig. 3) : $\tau\left(B_{s}^{0}\right)=$ $1.398 \pm 0.0044(\text { stat })_{-0.025}^{+0.028}($ sys $)$ ps. The result is in good agreement with previous experiments and the current world average value. The second analysis studied the decay chain $B_{s}^{0} \rightarrow J / \psi, J / \psi \rightarrow \mu^{+} \mu^{-}, \phi \rightarrow K^{+} K^{-}$ which gives rise to both $\mathrm{CP}$-odd and $\mathrm{CP}$-even final states. Therefore, a simultaneous unbinned maximum likelihood fit to the $B_{s}^{0}$ candidate mass, the pseudo-proper decay length and 3 decay angles describing the angular distribution of both $J / \psi$ and $\phi$ final states in transversity basis allows us to separate the two $\mathrm{CP}$ components and measure $\Delta \Gamma$ as illustrated in Fig. 4.With $0.8 \mathrm{fb}^{-1}$ and in the limit of no $\mathrm{CP}$ violation, the preliminary results illustrated in Fig. 4 yield to $\Delta \Gamma=0.15 \pm 0.10_{-0.04}^{+0.03} \mathrm{ps}^{-1}$ and the average $B_{s}^{0}$ lifetime: $\bar{\tau}\left(B_{s}^{0}\right)=1.53 \pm 0.08(\text { stat })_{-0.04}^{+0.01}($ sys $) p s$. The fitted $\mathrm{CP}$ violating interference term is consistent with no $\mathrm{CP}$ violation violation in the $\left(B_{s}^{0}, \bar{B}_{S}^{0}\right)$ system. 


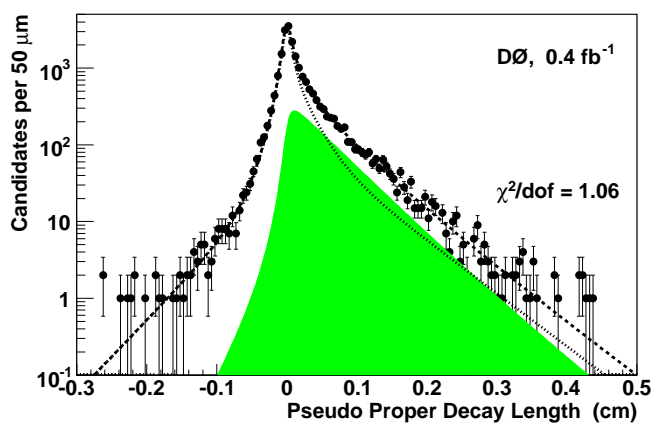

Figure 3. Pseudo-proper decay length distribution for $D_{s}^{-} \mu^{+}$candidates with the superimposed fit (dashed curve), combinatorial background (dotted curve) and $B_{s}$ signal (filled area).

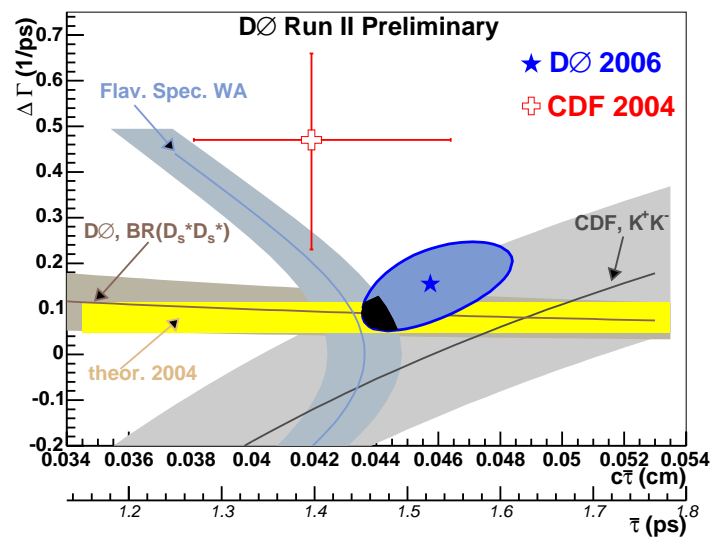

Figure 4. The 1- $\sigma$ (stat) contour for the three angle fit in $\Delta \Gamma$ vs $c \bar{\tau}$ plane compared to the 1- $\sigma$ band for the world average and the SM prediction (horizonthal band) ${ }^{2}$. Also shown are the CDF 2004 results $^{3}$ and the recent CDF measurement from $B_{s} \rightarrow K^{+} K^{-}$.

\section{References}

1. M. Di Pierro and E. Eichten, Phys. Rev. D64,(2001) 114004 [arXiv:hepph/0104208].

2. S. Eidelman et al, Particle Data Group, Phys. Lett. B 592, 1 (2004).

3. CDF Collaboration, Phys. Rev. Lett. 94, 101803 (2005). 